\title{
A Novel ELP2 Compound Heterozygous Mutation in a Boy with Severe Intellectual Disability, Spastic Diplegia, Stereotypic Behavior and Review of the Current Literature
}

\author{
Ayberk Turkyilmaz $^{\mathrm{a}} \quad$ Gunes Sager $^{\mathrm{b}}$

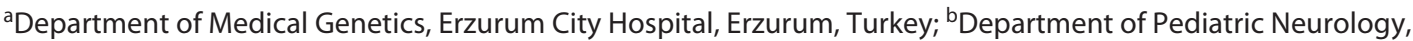 \\ Kartal Lutfi Kirdar City Hospital, Istanbul, Turkey
}

\section{Established Facts}

- ELP2 gene variations are associated with autosomal recessive mental retardation type 58 .

- ELP2-associated intellectual disability is a very rare neurodevelopmental phenotype.

\section{Novel Insights}

- Electroencephalogram abnormality is a novel feature of the ELP2-associated intellectual disability phenotype.

- The c.1189-1G>A variant is the first known splice-site variant in the ELP2 gene.

- The family presented here with a novel feature is the fourth such family described in the literature.

\section{Keywords}

Elongator complex $\cdot E L P 2 \cdot$ Intellectual disability $\cdot$ Novel mutation · Spastic diplegia · Stereotypic behavior $\cdot$ Wholeexome sequencing

\section{Abstract \\ The elongator complex consists of 6 highly conserved sub- unit proteins and is indispensable for various cellular func- tions, such as transcription elongation, histone acetylation, and tRNA modification. The elongator complex contains 2 subunits, each of which consists of 3 different proteins (en-}

coded by the ELP1-3 and ELP4-6 genes). According to the OMIM database, ELP2 gene variations have been reported to be associated with autosomal recessive mental retardation type 58 . Here, we report a male patient with severe intellectual disability, spastic diplegia, and stereotypic behavior; in addition, we also provide a review of the current literature. Using whole-exome sequencing analysis, we detected a novel compound heterozygous variation in the ELP2 gene. We present this case report to clarify the clinical findings of a very rare neurodevelopmental phenotype and to contribute new information to the current literature on genotypephenotype correlations.

C 2020 S. Karger AG, Base

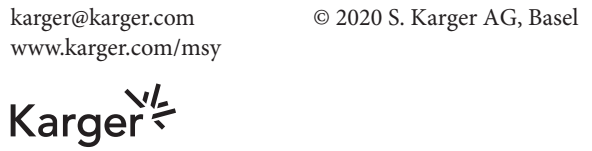

Ayberk Turkyilmaz

Department of Medical Genetics, Erzurum City Hospital

Cat Yolu Street, No. 2

Erzurum 25070 (Turkey)

ayberkturkyilmaz@gmail.com 


\section{Introduction}

The elongator complex consists of 6 highly conserved subunit proteins and is indispensable for various cellular functions such as transcription elongation, histone acetylation, tRNA modification, exocytosis, zygotic paternal DNA demethylation, sensitivity to DNA damaging agents, transcriptional silencing, and alpha-tubulin acetylation. The elongator complex comprises 2 subunits, each of which consists of 3 different proteins (encoded by the ELP1-3 and ELP4-6 genes) [Glatt and Müller, 2013].

According to the OMIM database, ELP2 gene variations have been reported to be associated with autosomal recessive mental retardation type 58 . Until now, the only homozygous mutation in the ELP2 gene reported in the literature was detected in 6 cases from 2 families investigated for intellectual disability [Najmabadi et al., 2011]. In addition, it has been reported that 2 brothers with severe intellectual disability, spastic diplegia, and behavioral problems had a compound heterozygous mutation in the ELP2 gene (Table 1) [Cohen et al., 2015].

\section{Case Report}

The male patient presented here was born at full term with a normal birth weight after an uneventful pregnancy to nonconsanguineous parents. His development was globally delayed from infancy on. The first concern was noted at age 3 months and was due to poor head control, hypotonicity, and lack of eye contact. The patient who was able to sit at 20 months can now crawl on his knees but cannot get up and walk. It is known that the boy, who is now 4 years old, cannot speak but makes sounds, has limited eye contact and object tracking, has no ability to mimic, and does not play with toys. Instead of pointing to his objects of interest, he "tells" what he wants by crying. He is able to chew and swallow food. The patient can join his hands in the midline and hold objects in the palm of his hand and pass them from one hand to the other, but he does not reach out to things on his own. The patient has severe intellectual disability with self-injurious behavior and temper tantrums.He was found to have language development consistent with 9 months of age, personal social development consistent with 3 months of age, fine motor skills consistent with 10 months of age, and gross motor skills consistent with 10 months of age in the Denver test performed at 3 years and 9 months. During follow-up, the patient showed improvement in terms of mental and motor development.

His head circumference was in the 50th percentile at birth, but later, acquired microcephaly was observed. In his anthropometric evaluation, height was $92 \mathrm{~cm}(-2.81 \mathrm{SDS})$, weight was $13 \mathrm{~kg}(-2.06$ SDS), and his body mass index was $15.36 \mathrm{~kg} / \mathrm{m}^{2}(-0.25 \mathrm{SDS})$. His physical examination revealed truncal hypotonia, lower limb spasticity, and diffuse hyperreflexia. Cerebellar and extrapyramidal signs were not detected. His ophthalmological and hearing examinations were normal, and he did not experience epileptic seizures.

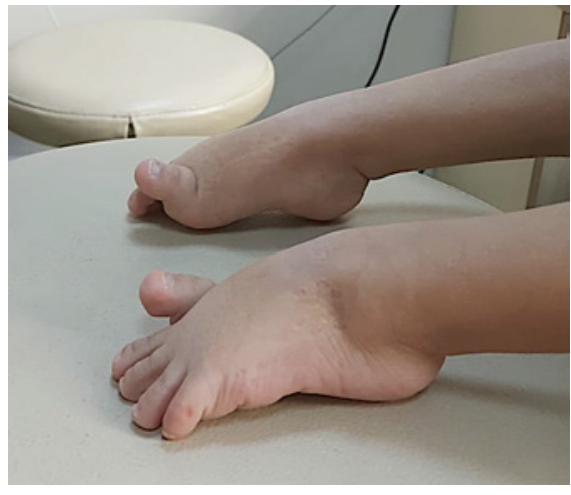

Fig. 1. Thin legs and hallux deformity.

According to his dysmorphology examination, he had very thin legs, hallux deformity, and no significant dysmorphic facial features (Fig. 1).

Abdominal ultrasonography, echocardiography, electromyography (EMG), and nerve conduction studies were found to be nor$\mathrm{mal}$, and there was no abnormality in magnetic resonance imaging (MRI) of the brain. Electroencephalogram (EEG) showed slowwave discharges located in the posterior regions with normal background (Fig. 2). Levetiracetam treatment was started for the disorder detected in the EEG, and 6 months later, the follow-up EEG was normal. The patient, who did not have any seizures while on levetiracetam treatment, was started on gabapentin therapy for lower limb spasticity.

Complete blood count, plasma creatine kinase, thyroid hormone, uric acid, and vitamin B12 levels were normal according to the laboratory investigations of the patient. In addition, plasma amino acids, serum lactate, plasma ammonia, plasma acylcarnitine profile, plasma very-long-chain fatty acid level, lysosomal enzyme screening, urine organic acid profile, urine glycosaminoglycan, and urine oligosaccharide assays that were conducted to investigate metabolic disorders were all normal. His karyotype was $46, X Y$. To identify any submicroscopic chromosomal aberrations, an array comparative genomic hybridization (aCGH) analysis was performed. The aCGH analysis was normal, $\operatorname{arr}(1-22) \times 2,(\mathrm{X}, \mathrm{Y}) \times 1$. According to whole-exome sequencing (WES) analysis, a novel heterozygous splice-site variant (NM_001242875: c.1189-1G>A) and a heterozygous missense variant (NM_001242875: c.1580G $>$ A, p.Arg527Gln) were identified in the ELP2 gene (Fig. 3). In the segregation analysis, a splice-site variant (NM_001242875: c.1189$1 \mathrm{G}>\mathrm{A}$ ) was detected in the patient's father, while a missense variant (NM_001242875: c.15801G>A, p.Arg527Gln) was detected in his mother. The present splice-site variant (NM_001242875: c.1189-G>A) was classified as pathogenic according to the American College of Medical Genetics and Genomics (ACMG) criteria [Richards et al., 2015]. This splice-site variant has not been previously reported in the Human Gene Mutation Database (http:// www.hgmd.cf.ac.uk/ac/index.php), the ClinVar archive, or in population studies (gnomAD, ESP, or 1000 Genome Project). The missense variant (NM_001242875: c.1580G>A, p.Arg527Gln) we detected was classified as likely pathogenic based on the ACMG criteria. The missense variant has been previously reported as like- 
Table 1. Summary of the phenotypic features of patients with variations in ELP2 in the literature

\begin{tabular}{|c|c|c|c|c|c|}
\hline & Present patient & $\begin{array}{l}\text { Family } 1 \text { (3 } \\
\text { affected children) } \\
\text { Najmabadi et al. } \\
\text { [2011] }\end{array}$ & $\begin{array}{l}\text { Family } 2 \\
\text { (3 affected children) } \\
\text { Najmabadi et al. } \\
\text { [2011] }\end{array}$ & $\begin{array}{l}\text { Family } 3 \text { (patient 1) } \\
\text { Cohen et al. [2015] }\end{array}$ & $\begin{array}{l}\text { Family } 3 \text { (patient 2) } \\
\text { Cohen et al. [2015] }\end{array}$ \\
\hline Gender & Male & NR & NR & Male & Male \\
\hline Age, years & 4 & NR & NR & 27 & 24 \\
\hline Gestation & NR & NR & NR & Term & Term \\
\hline Prenatal growth retardation & NR & NR & NR & - & - \\
\hline Hypotonia & + & NR & NR & + & + \\
\hline Intellectual disability & Severe & Mild & Severe & Severe & Severe \\
\hline Developmental regression & - & NR & NR & + & - \\
\hline Self-injurious behavior & + & NR & NR & + & + \\
\hline Aggression & + & NR & NR & + & + \\
\hline Lower extremity spasticity & + & NR & NR & + & + \\
\hline Speech & Never gained & NR & NR & Never gained & Never gained \\
\hline Motor development & Severely retarded & NR & NR & Severely retarded & Severely retarded \\
\hline Stereotypic behavior & + & NR & NR & + & + \\
\hline Eye examination & Normal & NR & NR & NR & NR \\
\hline Dysmorphic features & - & NR & NR & $\begin{array}{l}\text { Depressed and broad nasal } \\
\text { bridge, everted lower lip }\end{array}$ & $\begin{array}{l}\text { Depressed and broad nasal } \\
\text { bridge, everted lower lip, } \\
\text { broad nasal tip }\end{array}$ \\
\hline Additional features & Hallux deformity & NR & NR & - & - \\
\hline
\end{tabular}

NR, not reported; - absent; + present.

ly pathogenic in ClinVar. In the Genome Aggregation Database (gnomAD) version 2.1.1, the allele frequency of this variant is 0.00000867 , and it is considered rare because its minor allele frequency score is below $1 \%$. In silico analyses predicted the pathogenicity of this variant. All variants were confirmed by Sanger sequencing.

\section{Discussion}

In our case, we believe that the novel variations in the ELP2 gene are pathogenic and help explain the clinical features of this patient. The paternal splice acceptor site c.1189- 


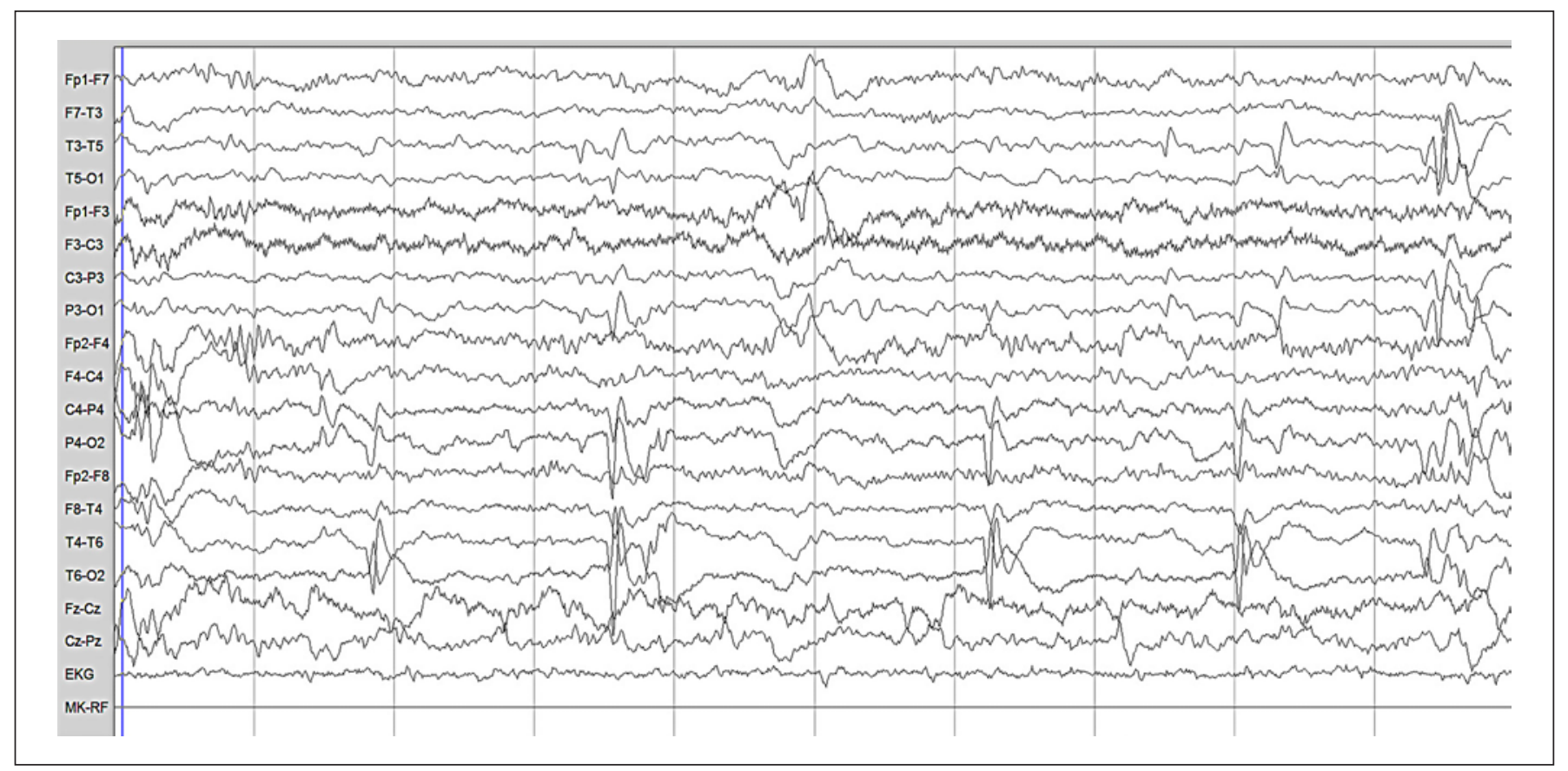

Fig. 2. Interictal EEG showing high-amplitude slow-wave spikes that emerged predominantly from occipital regions in both hemispheres and normal background rhythm (Sensitivity: $10 \mu \mathrm{V} / \mathrm{mm}$; Bandpass 0.5-70 Hz).

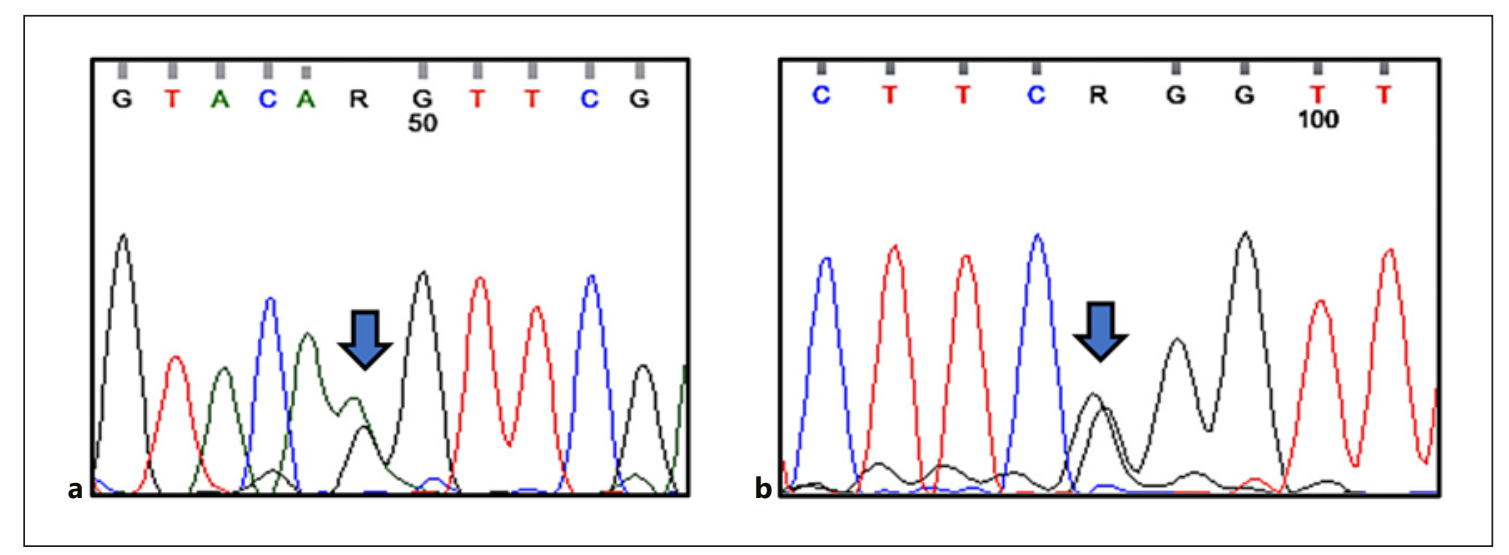

Fig. 3. a Paternal heterozygous c.1189-1G>A variant in intron 10 of the ELP2 gene. b Maternal heterozygous c.1580G $>$ A (p.Arg527Gln) variant in exon 14 of the ELP2 gene.

$1 \mathrm{G}>\mathrm{A}$ variation detected in intron 10 of the ELP2 gene is predicted to disrupt splicing according to an in silico analysis (Human Splicing Finder, MutationTaster). Moreover, this variation should be considered pathogenic once the frequency of the variant in population studies and the ACMG criteria are considered. Additionally, we predict that the maternal missense c.1580G >A (p.Arg527Gln) variation detected in exon 14 of the ELP2 gene is pathogenic when many criteria are evaluated together. First of all, 5 patients from 2 different families, who had similar clinical manifestations as in our patient, were also found to have a variation that caused another amino acid change in the same codon and this variation was considered pathogenic [Najmabadi et al., 2011; Cohen et al., 2015]. Second, segregation analysis was found to be consistent for affected/nonaffected individuals. Third, the position where the variation was detected is a preserved point between species in the evolutionary process. Fourth, this variation led to the transformation of po- 
lar basic amino acid into neutral amino acid. In addition, the fact that a variation was detected in codon 527 in 6 of 9 patients in the literature suggests that this region may be a hotspot in terms of mutation [Najmabadi et al., 2011; Cohen et al., 2015].

ELP2 gene alterations are considered in the differential diagnosis of intellectual disability and developmental delay. Cohen et al. [2015] reported 2 cases in a single family. Those patients had severe intellectual disability with spastic diplegia, choreoathetosis, and regression. They also had hypotonia and developmental delay that began in infancy (Table 1). Our case not only exhibited hypotonicity and global developmental delay that began in infancy, but also had severe intellectual disability with spastic diplegia. The boy also displayed self-injurious behavior, as mentioned in other cases. However, our case did not have extrapyramidal signs, and thus, a movement disorder should not be considered as the sole possibility in cases that feature ELP2 alterations. Our patient had a static clinical course without regression, which differed from that in cases reported by Cohen et al. [2015] (Table 1). Since static spastic diplegia is often associated with cerebral palsy, the patients can be followed up incorrectly for cerebral palsy because rare genetic syndromes are not considered despite normal MRI findings. Therefore, cases with normal MRI findings should be investigated for ELP2-related spastic diplegia

Dysmorphology examination revealed very slim legs and hallux deformity, which have not been previously reported. Although some dysmorphic facial features were noted in 2 siblings reported by Cohen et al. [2015]; our patient did not exhibit any significant dysmorphic features (Table 1).

The elongator complex consists of several protein subunits, and the ELP1, ELP2, and ELP4 genes have been associated with various neurological phenotypes according to the OMIM database. Variations in the ELP1 gene are responsible for an autosomal recessive disorder that results in the degeneration of the peripheral nervous system. The patient's leg structure was similar to that seen in congenital peripheral neuropathies. Therefore, an EMG study was performed in our patient, but the results were normal. ELP4 genetic variants are associated with benign rolandic epilepsy [Strug et al., 2009]. In our patient, slow-wave discharges were detected in posterior regions, but clinical seizures were not observed. Elongator complex genes share similar functions in transcription elongation, histone acetylation, and tRNA modification; these similarities may explain the EEG abnormalities observed in our patient.

So far, 9 cases have been reported from 4 different families with ELP2-related phenotypes. The low number of exist- ing cases and the absence of follow-up data make it difficult to comment on the long-term comorbidities, life expectan$\mathrm{cy}$, and causes of mortality. Clinical follow-up of this patient may provide additional relevant insight in the future.

We presented this case report to clarify the clinical findings of a very rare neurodevelopmental phenotype and to contribute additional information to the current literature on genotype-phenotype correlations.

\section{Statement of Ethics}

All experimental procedures were conducted in accordance with the principles of the Declaration of Helsinki, and written informed consent was obtained from the patient's parents.

\section{Conflict of Interest Statement}

The authors have no conflicts of interest to declare.

\section{Funding Sources}

There was no funding relevant to this study.

\section{Author Contributions}

A.T. performed the WES analysis as well as the segregation study and drafted the manuscript. G.S. conducted the patient's physical examination and critically reviewed the manuscript.

References

Cohen JS, Srivastava S, Farwell KD, Lu HM, Zeng $\mathrm{W}, \mathrm{Lu} \mathrm{H}$, et al. ELP2 is a novel gene implicated in neurodevelopmental disabilities. Am J Med Genet A. 2015;167(6):1391-5.

Glatt S, Müller CW. Structural insights into Elongator function. Curr Opin Struct Biol. 2013; 23(2):235-42.

Najmabadi H, Hu H, Garshasbi M, Zemojtel T, Abedini SS, Chen W, et al. Deep sequencing reveals 50 novel genes for recessive cognitive disorders. Nature. 2011;478(7367):57-63.

Richards S, Aziz N, Bale S, Bick D, Das S, GastierFoster J, et al. Standards and guidelines for the interpretation of sequence variants: a joint consensus recommendation of the American College of Medical Genetics and Genomics and the Association for Molecular Pathology. Genet Med. 2015;17(5):405-24.

Strug LJ, Clarke T, Chiang T, Chien M, Baskurt Z, $\mathrm{Li} W$, et al. Centrotemporal sharp wave EEG trait in rolandic epilepsy maps to Elongator Protein Complex 4 (ELP4). Eur J Hum Genet. 2009;17(9):1171-81. 